\title{
Pure single-site robot-assisted pyeloplasty with the da Vinci SP surgical system: Initial experience
}

\author{
Ji Eun Heo', Sung Ku Kang ${ }^{1}$, Dong Hoon Koh ${ }^{2}$, Joon Chae Na', Yong Seung Lee', Woong Kyu Han ${ }^{1}$, \\ Young Deuk Choi ${ }^{1}$, Won Sik Jang ${ }^{1}$ \\ 'Department of Urology and Urological Science Institute, Yonsei University College of Medicine, Seoul, '²epartment of Urology, Konyang University College of Medicine, \\ Daejeon, Korea
}

Purpose: Laparoendoscopic single-site surgery (LESS) reduces the limited invasiveness of conventional laparoscopy while providing superior cosmetic results. However, LESS remains a challenging surgical technique, even in robotic surgery, primarily due to the lack of triangulation and limited instrument movement. The da Vinci SP surgical system (Intuitive Surgical) was recently introduced to overcome these limitations. We describe our initial experience with pure single-site robot-assisted pyeloplasty (RAP) for ureteropelvic junction obstruction (UPJO) using the da Vinci SP surgical system.

Materials and Methods: Three consecutive patients who were diagnosed with UPJO underwent RAP with the da Vinci SP surgical system from December 2018 to February 2019 at our institution. The surgical technique involved reproducing the steps of multiport RAP. A 30-mm umbilical incision was made and the GeIPOINT was inserted. The multichannel robotic port and the assistant's port were placed through the GelSeal cap. In all patients, Anderson-Hynes dismembered pyeloplasty was performed. The ureteral double I stent was inserted antegrade, and the drain was not placed.

Results: The procedures were successfully completed using a pure single-site approach. There was no need for additional port placement or conversion to laparoscopic or open surgery. Total operative time in the three patients was 139, 180, and 213 minutes, respectively. No intraoperative complications occurred, and blood loss was minimal. The postoperative course of all patients was uneventful with no complications greater than Clavien-Dindo grade I surgical complications.

Conclusions: Pure single-site RAP using the da Vinci SP surgical system is feasible and safe.

Keywords: Laparoscopy; Robotic surgical procedures; Ureteral obstruction

This is an Open Access article distributed under the terms of the Creative Commons Attribution Non-Commercial License (http://creativecommons.org/licenses/by-nc/4.0) which permits unrestricted non-commercial use, distribution, and reproduction in any medium, provided the original work is properly cited.

\section{INTRODUCTION}

Laparoendoscopic single-site surgery (LESS) is a laparoscopic procedure performed through a single skin incision. This minimally invasive surgery was developed to minimize port-related complications, recovery time, and postoperative pain and to achieve superior cosmetic results [1,2]. Although LESS is feasible for several urologic procedures [3], the technique is highly challenging even for expert laparoscopic surgeons.

Received: 12 March, 2019 - Accepted: 19 April, 2019

Corresponding Author: Won Sik Jang

Department of Urology and Urological Science Institute, Yonsei University College of Medicine, 50-1 Yonsei-ro, Seodaemun-gu, Seoul 03722, Korea

TEL: +82-2-2228-2323, FAX: +82-2-312-2538, E-mail: sindakjang@yuhs.ac

ORCID: https://orcid.org/0000-0002-9082-0381 
Since Kaouk et al. [4] reported the first series of singleincision surgeries for the upper urinary tract using the da Vinci surgical system (Intuitive Surgical, Sunnyvale, CA, USA), the use of robotic single-site surgery for urologic problems has been globally reported [5,6]. However, intracorporeal suturing and traction are difficult and technically time-consuming because of clashing of the external robotic instruments and the loss of triangulation $[7,8]$. These challenges are occasionally overcome by the additional placement of an assistant port [9]

The da Vinci SP surgical system was developed as a novel robotic platform for successfully performing "pure" robotic single-site surgery while overcoming the aforementioned difficulties [10]. The system recently received clearance from the Food and Drug Administration [11]. The da Vinci SP surgical system was introduced for the first time in Korea at the end of 2018.

Here, we describe our experience with our first three cases of robot-assisted pyeloplasty (RAP) performed using the da Vinci SP surgical system in Severance Hospital. We report on the technical feasibility and perioperative outcomes. To the best of our knowledge, this is the first case series on RAP performed using the da Vinci SP surgical system in Asia.

\section{MATERIALS AND METHODS}

\section{Patients}

Three consecutive patients diagnosed with ureteropelvic junction obstruction (UPJO) underwent RAP performed by use of the da Vinci SP surgical system from December 2018 to February 2019. The surgeries were done by a single surgeon (W.S.J) who had performed eight cases of conventional RAP in a recent year at our institution,

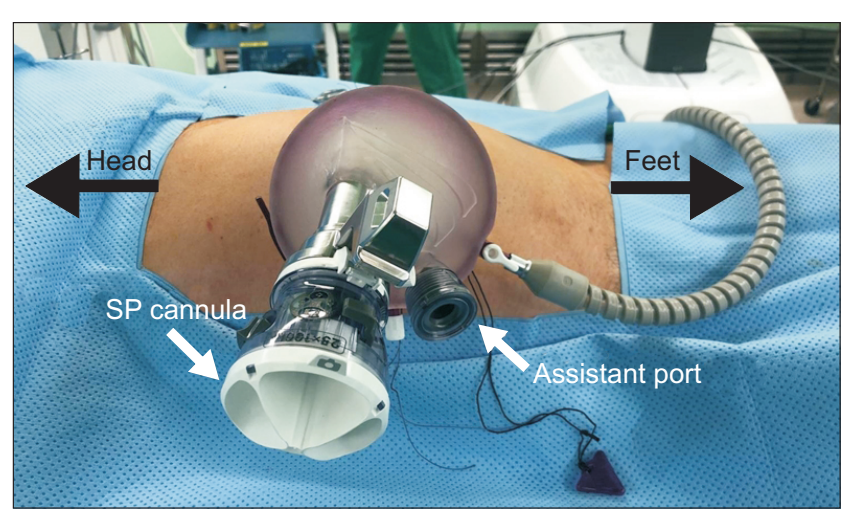

Fig. 1. Placement of the GelPOINT advanced access platform. A 25-mm multichannel port and a $12-\mathrm{mm}$ assistant port were placed through the GelSeal cap. which is a high-volume robotic center. All patients reported flank pain on the affected side due to UPJO and signed an informed consent after the novel robotic system had been explained to them. This study was approved by the Institutional Review Board (IRB) of Severance Hospital (IRB approval number: 1-2019-0003).

\section{Surgical procedure}

RAP was performed with the da Vinci SP surgical system by using the dismembered technique with a transperitoneal approach. Patients were positioned in a semilateral position. A 30-mm umbilical incision was made to reach the peritoneal cavity, and the GelPOINT advanced access platform (Applied Medical, Rancho Santa Margarita, CA, USA) was inserted (Fig. 1). A 25-mm multichannel port containing an articulating robotic camera and two double-jointed robotic instruments and a 12-mm port for an assistant's laparoscopic instrument were placed through the GelSeal cap (Fig. 2).

The surgical technique involved reproducing the steps of our own multi-port RAP. An incision was made along the white line of Toldt, and the colon was mobilized medially to expose Gerota's fascia; an incision was made in the Gerota's fascia to isolate the renal pelvis and ureter. Pyeloplasty was performed using the Anderson-Hynes technique. After excision of the obstructed ureteropelvic junction, the ureter was longitudinally spatulated. Anastomosis was performed on the posterior side with a running 4-0 absorbable, sterile suture and a double $J$ stent was inserted antegrade. Once the stent was correctly placed, anastomosis was completed on the anterior side (Video clip, Supplementary material). A surgical drain was not inserted. Layered wound closure was

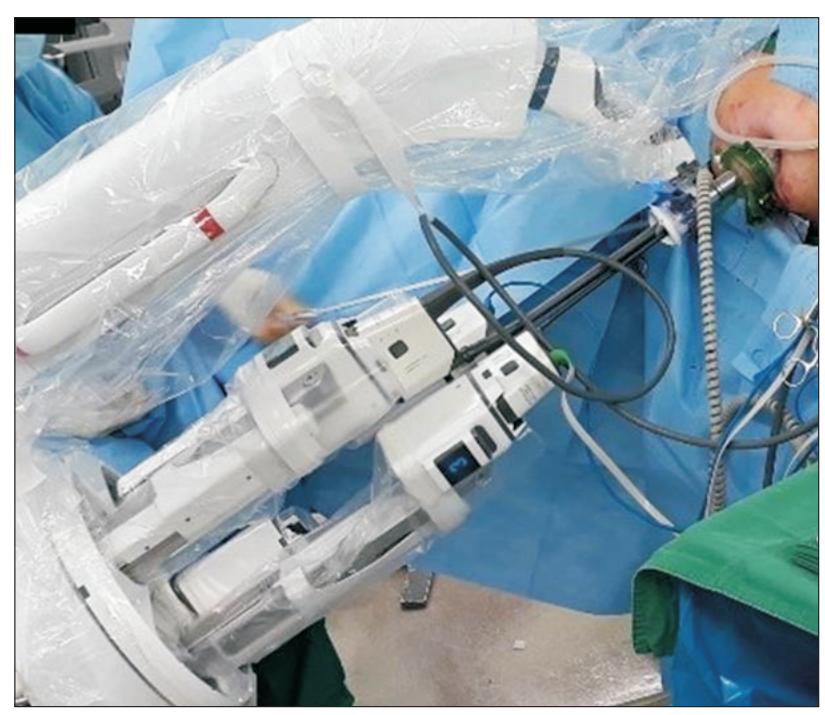

Fig. 2. Placement of the instruments and docked single-port platform. 
performed, and the skin was closed with a skin bond.

Preoperative variables, intraoperative surgical outcomes, and immediate postoperative complications were investigated. The Clavien-Dindo classification was used to grade the severity of postoperative complications [12]

\section{RESULTS}

The procedures were successfully completed using a pure single-site approach. There was no need for additional port placement or conversion to laparoscopic or open surgery. The patients' demographic characteristics and perioperative data are shown in Table 1. Total operative time in the three patients was 139, 180, and 213 minutes, respectively. The third patient had a large parapelvic cyst and had undergone endopyelotomy with balloon dilation 3 years ago; marsupialization of the parapelvic cyst was performed at the same time, and there was a moderate adhesion near the uteropelvic joint. No intraoperative complications occurred, and blood loss was minimal. The postoperative course of all patients was uneventful with no complications greater than Clavien-Dindo grade I postoperative surgical complications. The cosmetic result was satisfactory (Fig. 3).

\section{DISCUSSION}

We have validated the feasibility of pure single-site RAP performed using the da Vinci SP surgical system. For pyeloplasty, an elongated skin incision is not required to collect the specimen and there are no oncologic risks associated with the procedure; thus, pyeloplasty is an option for suitable single-site surgery. Despite the technical difficulties associated with the procedures, single-site laparoscopic and robotic pyeloplasty have frequently been performed owing to the need for adequate cosmesis $[6,7,13]$. The main concern in single-site surgery is the loss of triangulation [7,8]; however, technologic advancements have aided in overcoming this problem.

The da Vinci SP surgical system was designed after development of the SP 999 and SP 1098 prototypes [14]. The da Vinci SP surgical system includes three doublejointed wristed instruments and a fully wristed three-

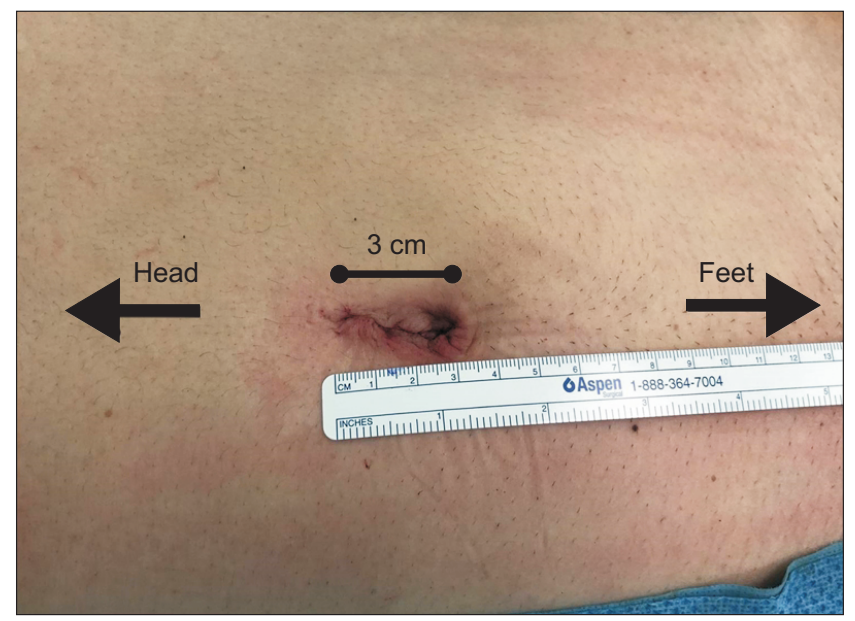

Fig. 3. Cosmetic result after skin closure.

Table 1. Patients' characteristics and perioperative data

\begin{tabular}{|c|c|c|c|}
\hline Characteristic & Case no. 1 & Case no. 2 & Case no. 3 \\
\hline Age (y) & 42 & 30 & 71 \\
\hline Sex & Male & Male & Female \\
\hline Affected side & Left & Right & Left \\
\hline Body mass index $\left(\mathrm{kg} / \mathrm{m}^{2}\right)$ & 22.49 & 27.08 & 18.82 \\
\hline Preoperative eGFR $\left(\mathrm{mL} / \mathrm{min} / \mathrm{m}^{2}\right)$ & 91 & 108 & 43 \\
\hline Grade of UPJO & High & Low & High \\
\hline Operative time $(\mathrm{min})$ & 139 & 180 & 213 \\
\hline Docking time (min) & 5 & 10 & 10 \\
\hline Console time (min) & 118 & 145 & 185 \\
\hline Suture time (min) & 29 & 47 & 53 \\
\hline Intraoperative complications & No & No & No \\
\hline Conversion to other surgery & No & No & No \\
\hline Estimated blood loss (mL) & Minimal & Minimal & 50 \\
\hline Discharge & POD 4 & POD 3 & POD 3 \\
\hline Postoperative eGFR $\left(\mathrm{mL} / \mathrm{min} / \mathrm{m}^{2}\right)$ & 105 & 113 & 81 \\
\hline Postoperative complications & No & No & No \\
\hline Pain at discharge, NPIS score & 2 & 2 & 3 \\
\hline
\end{tabular}

eGFR, estimated glomerular filtration rate; UPJO, ureteropelvic junction obstruction; POD, postoperative day; NPIS, numerical pain intensity scale. 
dimensional camera. This double-jointed instrument, called the "EndoWrist," performs an "elbow" movement to provide triangulation. The double-jointed instruments, the camera, and the assistant's 5-mm laparoscopic instrument are placed through a single multichannel port. The single arm enables $360^{\circ}$ anatomic access.

Although the EndoWrist instrument provides elbow movement for achieving intracorporeal triangulation, the angle formed on curving the EndoWrist instrument is less than $90^{\circ}$, making proper spatulation of the ureter difficult to perform. Anastomotic suturing is not difficult. Moreover, the assistant experiences difficulty because of the limited working space, which is common with single-site surgery, and good coordination between the surgeon and the assistant is required. The surgeon should move the camera or instruments when the assistant's instrument enters into the operation field for traction or suction. Therefore, an additional assistant port could be inserted in cases of high risk of bleeding or when many assists are required. Although an additional port was not used in the three cases described in this report, we inserted an additional port to use an EndoStapler for robot-assisted radical nephroureterectomy using the da Vinci SP surgical system.

There were some limitations to this study: namely, the small sample size and short follow-up duration. When the cases of conventional RAP performed by the same surgeon (W.S.J.) in a recent 1-year period were reviewed, the mean operative time was $136 \pm 53.0$ minutes and the mean suture time was $38 \pm 9.0$ minutes. If the surgeon becomes accustomed to performing surgery using the da Vinci SP surgical system, the operative time or suture time could be shortened by as much as conventional RAP. The functional outcomes of RAP using da Vinci SP surgical system are not yet confirmed because the imaging studies will be performed after removal of the double $\mathrm{J}$ stent 3 months after surgery according to our hospital protocol. However, the results of this preliminary study are promising. There were no cases of conversion or complications, which showed the feasibility and safety of pure single-site RAP with the da Vinci SP surgical system. Studies analyzing more patients and long-term outcomes are needed. To confirm our results, the treatment outcomes of RAP performed using da Vinci SP surgical system should be compared with those of conventional RAP or LESS pyeloplasty.

\section{CONCLUSIONS}

Pure single-site RAP using the da Vinci SP surgical system is feasible and safe. Further studies involving more patients and long-term outcomes are needed to confirm our results.

\section{CONFLICTS OF INTEREST}

The authors have nothing to disclose.

\section{SUPPLEMENTARY MATERIAL}

The supplementary video clip can also be accessed by scanning a QR code, or will be available on YouTube: https:// youtu.be/8WVgnxFLpAM.

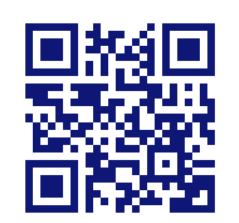

\section{REFERENCES}

1. Gettman MT, Box G, Averch T, Cadeddu JA, Cherullo E, Clayman RV, et al. Consensus statement on natural orifice transluminal endoscopic surgery and single-incision laparoscopic surgery: heralding a new era in urology? Eur Urol 2008;53:111720 .

2. Box G, Averch T, Cadeddu J, Cherullo E, Clayman R, Desai $\mathrm{M}$, et al.; Urologic NOTES Working Group. Nomenclature of natural orifice translumenal endoscopic surgery (NOTES) and laparoendoscopic single-site surgery (LESS) procedures in urology. J Endourol 2008;22:2575-81.

3. Autorino R, Cadeddu JA, Desai MM, Gettman M, Gill IS, Kavoussi LR, et al. Laparoendoscopic single-site and natural orifice transluminal endoscopic surgery in urology: a critical analysis of the literature. Eur Urol 2011;59:26-45.

4. Kaouk JH, Goel RK, Haber GP, Crouzet S, Stein RJ. Robotic single-port transumbilical surgery in humans: initial report. BJU Int 2009;103:366-9.

5. Han WK, Kim DS, Jeon HG, Jeong W, Oh CK, Choi KH, et al. Robot-assisted laparoendoscopic single-site surgery: partial nephrectomy for renal malignancy. Urology 2011;77:612-6.

6. Olweny EO, Park SK, Tan YK, Gurbuz C, Cadeddu JA, Best SL. Perioperative comparison of robotic assisted laparoendoscopic single-site (LESS) pyeloplasty versus conventional LESS pyeloplasty. Eur Urol 2012;61:410-4.

7. Best SL, Donnally C, Mir SA, Tracy CR, Raman JD, Cadeddu JA. Complications during the initial experience with laparoendoscopic single-site pyeloplasty. BJU Int 2011;108:1326-9.

8. Autorino R, Kaouk JH, Stolzenburg JU, Gill IS, Mottrie A, Tewari A, et al. Current status and future directions of robotic 
single-site surgery: a systematic review. Eur Urol 2013;63:26680 .

9. Seideman CA, Tan YK, Faddegon S, Park SK, Best SL, Cadeddu JA, et al. Robot-assisted laparoendoscopic single-site pyeloplasty: technique using the da Vinci Si robotic platform. J Endourol 2012;26:971-4.

10. Kaouk J, Garisto J, Eltemamy M, Bertolo R. Pure single-site robot-assisted partial nephrectomy using the SP surgical system: initial clinical experience. Urology 2019;124:282-5.

11. Kaouk J, Bertolo R, Eltemamy M, Garisto J. Single-port robotassisted radical prostatectomy: first clinical experience using the SP surgical system. Urology 2019;124:309.

12. Dindo D, Demartines N, Clavien PA. Classification of surgical complications: a new proposal with evaluation in a cohort of 6336 patients and results of a survey. Ann Surg 2004;240:20513.

13. Sorokin I, Cadeddu JA. Robotic laparoendoscopic single site: pyeloplasty and nephrectomy chapter. J Endourol 2018;32:S8892.

14. Bertolo R, Garisto J, Gettman M, Kaouk J. Novel system for robotic single-port surgery: feasibility and state of the art in urology. Eur Urol Focus 2018;4:669-73. 